\title{
Clinical Experience of Sturdy Elevation of the Reconstructed Auricle
}

\author{
Jeong-Hwan Choi, \\ Ju-Chan Kim, \\ Min-Su Kim, \\ Myung-Hoon Kim, \\ Keun-Cheol Lee, \\ Seok-Kwun Kim \\ Department of Plastic and Reconstructive \\ Surgery, Dong-A University School of \\ Medicine, Busan, Korea
}

No potential conflict of interest relevant to this article was reported.

\begin{abstract}
Background: The ear is composed of elastic cartilage as its framework, and is covered with a thin layer of skin. Auricular reconstruction using autogenous cartilage in microtia patients requires delicacy. This paper reports clinical experiences related to elevation of reconstructed ear in the last 11 years.

Methods: This study was based on 68 congenital microtia patients who underwent auricular elevation in our hospital. Among these 68 patients, 47 patients were recruited. We compared the differences in the ear size, auriculocephalic angle, and conchal depth with those in the opposite ear, and the patients' satisfaction levels were investigated using a survey.

Results: The difference in the sizes of the two ears was less than or equal to $5 \mathrm{~mm}$ in 32 patients, 5 to $10 \mathrm{~mm}$ in 10 patients, and greater than or equal to $10 \mathrm{~mm}$ in 5 patients. The difference in the auriculocephalic angles of the two ears was less than or equal to 10 degrees in 14 patients, 10 to 20 degrees in 26 patients, and greater than or equal to 20 degrees in 7 patients. The difference in the conchal depths of the two ears was less than or equal to $5 \mathrm{~mm}$ in 24 patients, 5 to $10 \mathrm{~mm}$ in 19 patients, and greater than or equal to $10 \mathrm{~mm}$ in 4 patients. The average grade of 3.9 points out of 5 points was obtained by the patients with satisfactory surveys.

Conclusion: We could make enough protrusion and maintain the three-dimensional shape for a long time to satisfy our patients.
\end{abstract}

Keywords: Ear auricle / Cartilage / Transplantation

\section{INTRODUCTION}

The ear has a very sophisticated structure. It is composed of elastic cartilage as its framework, and is covered with a thin layer of skin. Ideally reconstructed ears have a good shape, adequate protrusion, and a symmetric location. Reconstructed ears are still thick and stiff, however, and they do not have a delicate three-dimensional structures as real normal ears do, when they are closely observed [1-3]. Auricular reconstruction using autogenous cartilage

\footnotetext{
Correspondence: Seok-Kwun Kim

Department of Plastic and Reconstructive Surgery, Dong-A University School of Medicine, 26 Daesingongwon-ro, Seo-gu, Busan 602-715, Korea

E-mail: sgkim1@dau.ac.kr

*This study was supported by research funds from Dong-A University.

Received February 10, 2014 / Revised February 24, 2014 / Accepted March 4, 2014
}

in microtia patients requires delicacy and artistic skill. To meet these demands, different reconstruction methods have been suggested, with a single stage operation to 4-stage operation $[2,4,5]$.

The two-stage reconstruction method was performed in this study. This method was shown better results than another technique in aspect of economic and cosmetic. In the first stage operation, the ear lobule was translocated to its normal location and a framework made of costal cartilages was inserted in the subcutaneous pocket of the area symmetrical to the opposite ear. Six months after the costal cartilage insertion, auricular elevation was performed in the second stage operation. When this two-stage method was used, auricular elevation in the second stage is important to the delicate shape of reconstructed auricle and maintaining adequate projection for a long time. We would introduce 
our experience of sturdy elevation of the reconstructed auricle and literature reviews are reported in this paper.

\section{METHODS}

\section{Patients}

This study was based on 68 congenital microtia patients who had undergone auricular elevation in this hospital from January 2000 to December 2011. Among these 68 patients, 47 who could be observed after over 1 year follow-up were recruited.

\section{Elevation technique}

In the first stage operation, costal cartilages for the auricular framework and block were harvested. The block of cartilage was to be used for the auricular elevation in the second stage operation. And the use of this cartilage for the auricular elevation was buried under the subcutaneous tissue in the donor site. The auricular elevation was performed in the second stage operation after 6 months. The cartilage that was buried in the donor site was taken out. An incision was made $5 \mathrm{~mm}$ behind the framework margin, and the framework was elevated above the level of the superficial temporal fascia with attention to avoid exposure of cartilage framework. The cartilage was carved to form a crescent shape with flat bottom and to get an enough height, and scarring or atrophy of reconstructed ear should be considered to making height of cartilage, so the projection of the reconstructed ear was symmetrical to the opposite normal or previously elevated ear (Fig. 1). Then block of cartilage was located at the posterior auricular sulcus. This cartilage was fixed using 4-0 nylon sutures so that it would not fall off. The bottom parts were fixed up to the fascia and the periosteum, and the upper part was firmly fixed at the framework in two points. And then, the cartilage was covered by an elevated rectangular mastoid fascial flap in the retro-auricular area at the level of deep mastoid fascia, and was fixed to the framework again using 4-0 nylon sutures with the elevated fascial flap (Figs. 2, 3). The raw surface for the skin graft was minimized by advancing the retro-auricular skin, and then the area was covered with a full-
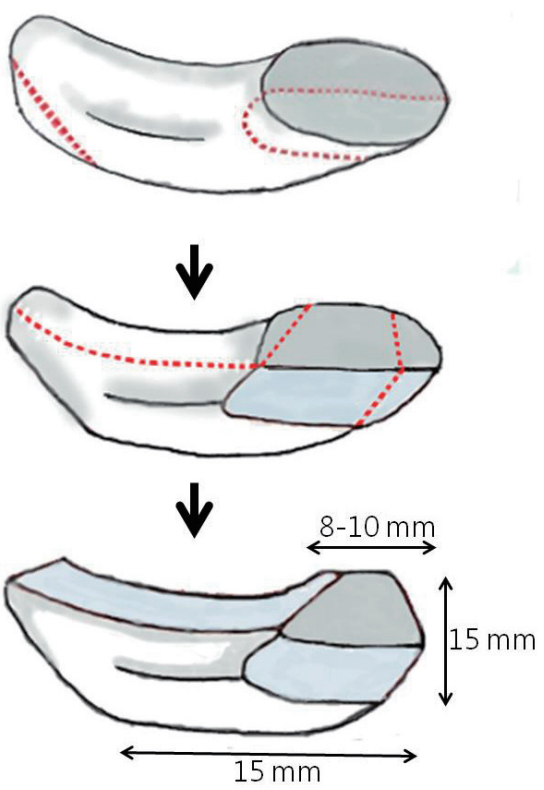

Fig. 1. Illustration of carving cartilage block.

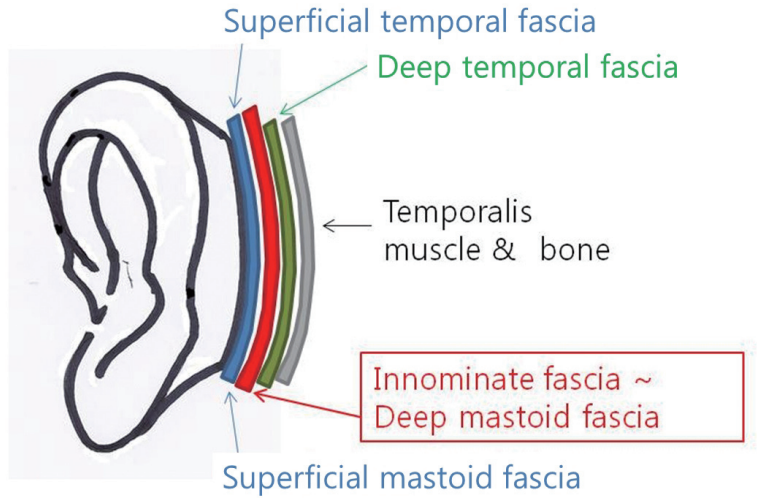

Fig. 2. Illustration of the anatomy of the retroauricular area.

thickness skin graft, which was harvested from the inguinal area. And the tie-over dressing was performed. If the depth of the helical sulcus was insufficient depth at the first operation or shallow depth after the elevation, the helical cartilage of helix was trimmed and sutured to the scapha. Through and through sutures with tie-over bolster dressing were performed for maintenance of the helical sulcus.

\section{Evaluation}

The ear size (length between the lowest part of the lobule and the most distant part of the helix), auriculocephalic angle (difference 
between the direction of two lines that come together from the root of the helix to the most lateral edge of the helix and the mastoid plane), and conchal depth (length between the antihelix and the concha) were observed in both ears, and the patients' satisfaction levels were investigated using a survey. Depth of helical sulcus was excluded because of correction during operation.

A difference in the sizes of the both ears that was less than or equal to $5 \mathrm{~mm}$ was categorized as excellent; 5 to $10 \mathrm{~mm}$, acceptable; and greater than or equal to $10 \mathrm{~mm}$, poor. A difference in the auriculocephalic angles of both ears that was less than or equal to 10 degrees was categorized as excellent; 10 to 20 degrees, acceptable; and greater than or equal to 20 degrees, poor. A difference in the conchal depths of both ears that was less than or equal to 5 $\mathrm{mm}$ was categorized as excellent; 5 to $10 \mathrm{~mm}$, acceptable; and greater than or equal to $10 \mathrm{~mm}$, poor.

The survey on the subjective satisfaction of patients with the degree of the ear projection and ear shape after elevation had a 5-point scale, with 5 meaning the respondent was very satisfied; 4 , satisfied; 3, neither satisfied nor unsatisfied; 2, unsatisfied; and 1, very unsatisfied (Figs. 4-7). We gave questionnaires to patient that follow up our hospital. When some patients had replied several times, the score of the latest last questionnaires was selected.

\section{RESULTS}

The patients were 7 to 26 years old at the time of the first-stage op- eration. There were more male patients (male to female ratio, 31:16), and more patients had microtia in their right ear (right to left ratio, 27:15) while five patients had microtia in both ears. The observation period was 1.5 to 10.3 years, and averaged 5.9 years.

The ear size was measured the length of a straight line between the highest area of helix and the lowest area of lobule. The ear size was $60.2 \pm 3.72 \mathrm{~mm}$ in the normal ear and $58.5 \pm 4.25 \mathrm{~mm}$ in the reconstructed ear. The difference in the sizes of both ears was less than or equal to $5 \mathrm{~mm}$ in 32 patients $(68 \%), 5$ to $10 \mathrm{~mm}$ in $10 \mathrm{pa}-$

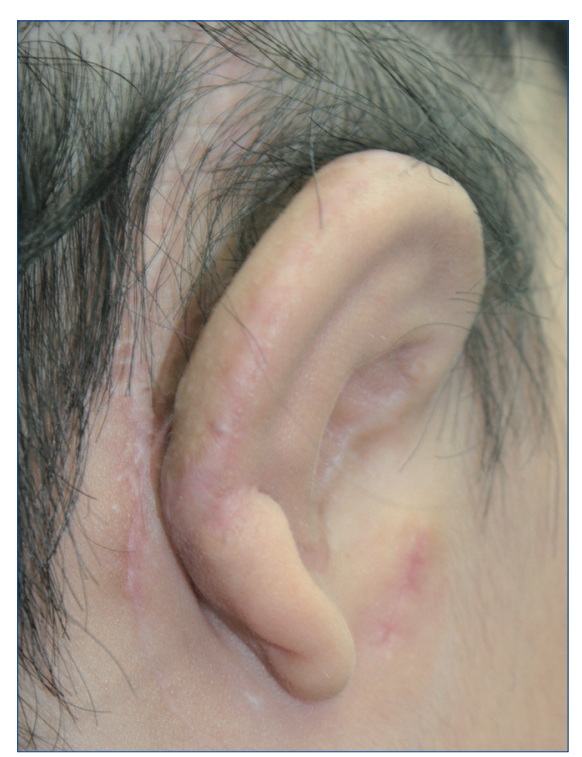

Fig. 4. Subjective satisfaction of patients after elevation. An 18-yearold girl who underwent auricular reconstruction 9.5 years ago. Her score of last questionnaires about satisfaction of was 2 .
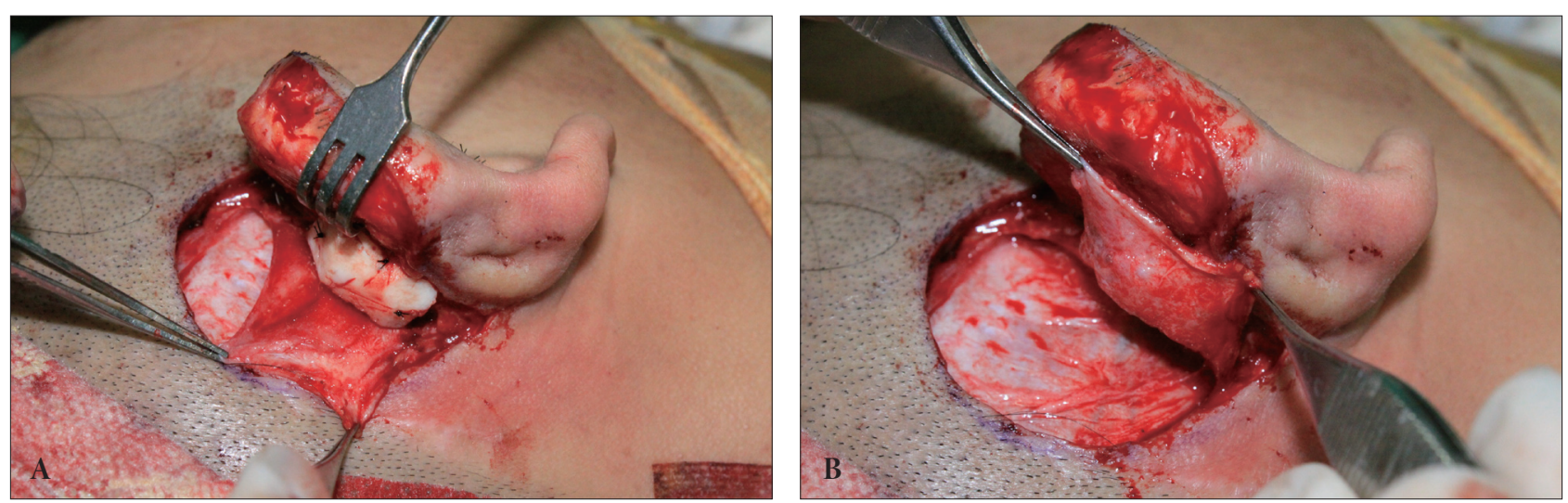

Fig. 3. Intraoperative views of the auricular elevation. (A) Carved cartilage for the support of the auricular framework. The cartilage was fixed using 4-0 nylon sutures to prevent it's falling off. (B) It was covered by the rectangular deep mastoid fascial flap in the retro-auricular area, and was fixed to the framework using 4-0 nylon sutures. 

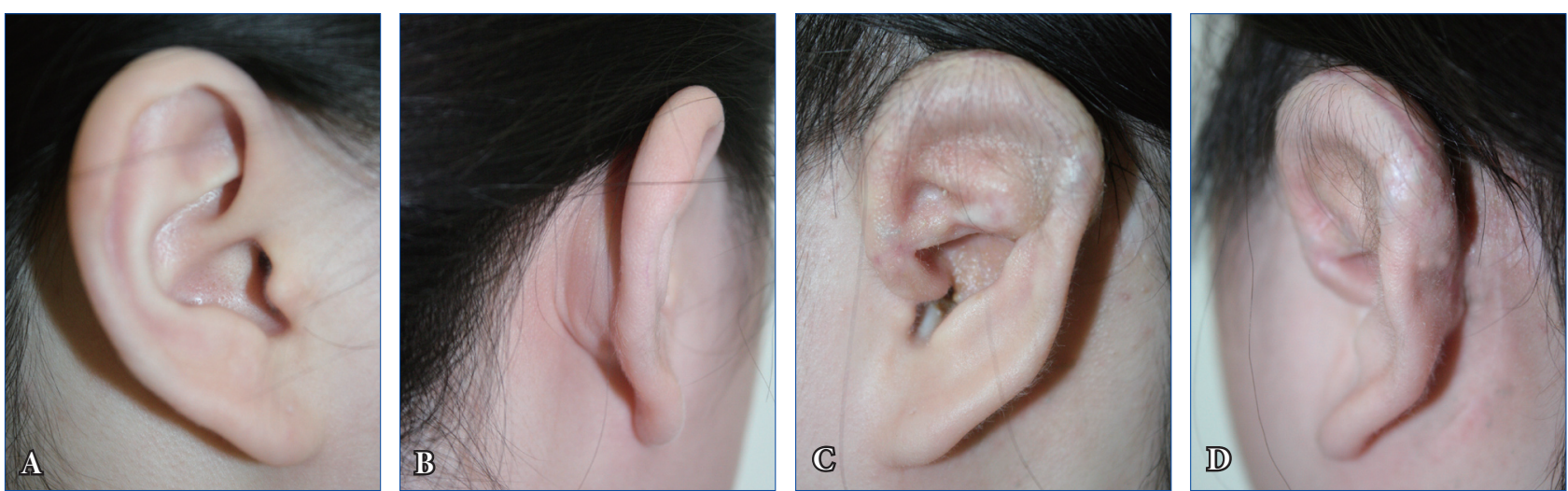

Fig. 5. Case 1. Post-elevation state of the auricle. A 14-year-old girl who underwent auricular reconstruction 3 years ago. She has an excellent ear size, auriculocephalic angle, and conchal depth. (A, B) Lateral and three-quarter views of the normal ear. (C, D) Lateral and three-quarter views of the reconstructed ear. Her score of last questionnaires about satisfaction was 5.
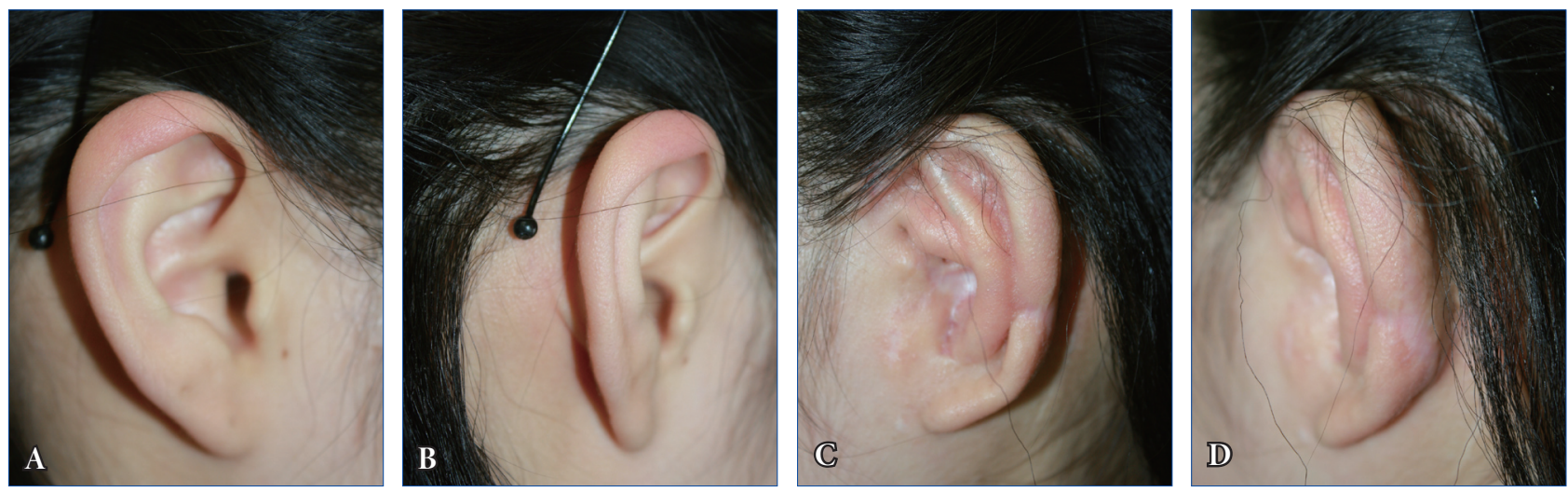

Fig. 6. Case 2. Post-elevation state of the auricle. A 15-year-old boy who underwent auricular reconstruction 8 years ago. He has an excellent ear size, auriculocephalic angle, and conchal depth. (A, B) Lateral and three-quarter views of the normal ear. (C, D) Lateral and three-quarter views of the reconstructed ear. His score of last questionnaires about satisfaction was 4 .
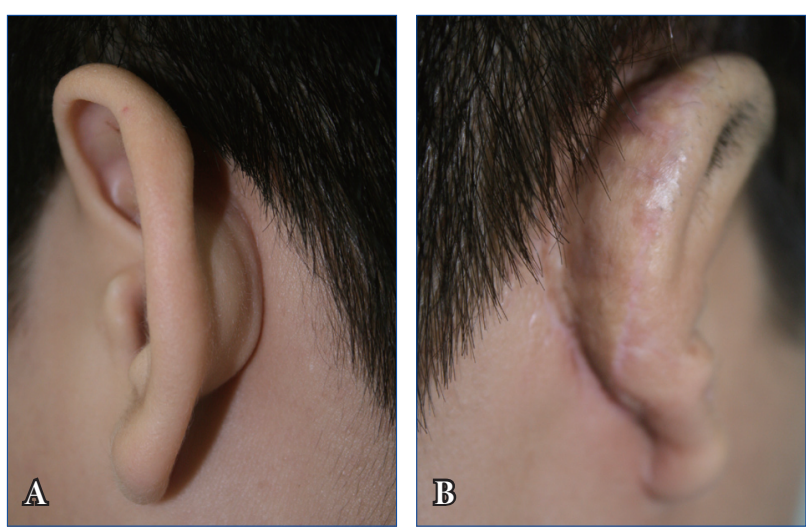

Fig. 7. Case 3. Post-elevation state of the auricle. A 17-year-old boy who underwent auricular reconstruction 3 years ago. He has an excellent ear size, acceptable auriculocephalic angle, and acceptable conchal depth. (A) Three-quarter view of the normal ear. (B) Threequarter view of the reconstructed ear. His score of last questionnaires about satisfaction was 3 . tients (21\%), and greater than or equal to $10 \mathrm{~mm}$ in 5 patients (11\%). The auriculocephalic angle was $45.1 \pm 10.4$ degrees in the normal ear and 39.6 \pm 7.1 degrees in the reconstructed ear. The difference in the auriculocephalic angles of both ears was less than or equal to 10 degrees in 14 patients (30\%), 10 to 20 degrees in $26 \mathrm{pa}$ tients $(55 \%)$, and greater than or equal to 20 degrees in 7 patients $(15 \%)$. The conchal depth of the ear was $18.9 \pm 1.8 \mathrm{~mm}$ in the normal ear and $16.5 \pm 2.6 \mathrm{~mm}$ in the reconstructed ear. The difference in the conchal depths of both ears was less than or equal to $5 \mathrm{~mm}$ in 24 patients (51\%), 5 to $10 \mathrm{~mm}$ in 19 patients (40\%), and greater than or equal to $10 \mathrm{~mm}$ in 4 patients (9\%) (Table 1, Figs. 5-7). The complications of operation were mild decreasing of projection by partial absorption of cartilage block and contracture of full-thickness skin graft (FTSG) (3 patients, 6\%), shallow auricular sulcus (6 
Table 1. Evaluation of morphometric study of the reconstructed ear

\begin{tabular}{lccc} 
Evaluation & Ear size & Auriculocephalic angle & Conchal depth \\
Excellent & $32(68)$ & $14(30)$ & $24(51)$ \\
Acceptable & $10(21)$ & $26(55)$ & $19(40)$ \\
Poor & $5(11)$ & $7(15)$ & $4(9)$ \\
\hline
\end{tabular}

Values are presented as number (\%).

patients, 13\%), delayed wound healing by infection or dehiscence (4 patients, $8 \%$ ), and widened scar of donor site (4 patients, $8 \%$ ). An average grade of 3.9 points was obtained by the patients with the satisfactory surveys, which showed that most of the patients were relatively satisfied.

\section{DISCUSSION}

It is very difficult to define a normal ear. Since the ear has constant anatomical landmarks such as the helix, scapha, antehelix, triangular fossa, concha, and tragus, it seems as if a normal ear can be defined when the ear is seen from the lateral side. However, many factors affect the normality of ears, such as their projecton, degree of folding, relative size, depth, and angles [6]. Nevertheless, since the ear is not located in the middle of the face, it does not draw attention unless it is too unnatural. Thus, the purpose of microtia reconstruction is to make an ear that does not look too awkward, so that others will not notice it [7]. Thus, when reconstructing the auricle, more focus should be given to the big contour, location, and degree of the ear projection rather than to detailed factors.

The following are important factors of elevation of auricle. First, there should be enough projection, and it should be maintained for a long time without deformation. Second, the blood circulation between the reconstructed structure and the elevated ear should not be disturbed. Third, the shape of the ear when it is viewed from the front should be maintained without abnormality after the elevation. Fourth, there should be no hair in the upper part of the elevated ear. Fifth, the number of surgery procedures should be minimized [8].

Many methods of auricle elevation have been introduced to meet these purposes. Steffensen [9] used a retro-auricular skin transposition flap to cover the sulcus, and Rueckert et al. [10] sug- gested a technique that uses tunnel formation. Alexander [11] also reported a method of elevation that uses a fascial flap. Maintaining a stable auriculocephalic angle using only soft tissue to support the auricle is difficult, however, which emphasizes the need for hard tissue to support the concha.

Brent [12] performed limited elevation of the auricle by heightening and exaggerating the helical rim. Nagata [8] used the temporoparietal fascial flap to wrap a semilunar piece of cartilage, which can create a fine firm projection. Ou et al. [13] reported the use of a retro-auricular fascial flap instead of a temporoparietal fascial flap to wrap a cartilage wedge. Prantl et al. [14] reconstructed the post-auricular sulcus using a rotation flap elevated from the neck.

The cartilage that would be used for the auricular elevation in our operation was the semilunar wedge shaped to be made big and tall enough to support the framework. As in the reports of $\mathrm{Ou}$ et al. [13] and Yoshimura et al. [15], the cartilage was covered using a superficial fascial flap in the retro-auricular area. In this process, the cartilage was fixed in more than 4 points with 4-0 nylon sutures. It is very important that the cartilage was fixed firmly and stably so that it would not fall off or move.

Lim et al. [16] reported that a retro-auricular fascial flap is less reliable than a temporoparietal fascia, because the dissection plane in the retro-auricular fascia is difficult to distinguish anatomically and an inevitable depression can be made by exposing the perioteum and the tendinous tissue in the mastoid site by elevating the retro-auricular fascial flap. Our deep mastoid fascial flap is similar to the retro-auricular fascial flap in the study of Lim.

One or two supporting cartilage were placed on retroauricular sulcus and fixed with 4-0 nylon sutures firmly to the periosteum and cartilage framework in 4 points. This supporting cartilage should be carved to form a crescent shape with flat bottom for prevention it's fall off.

Deep mastoid fascial flap must be enough large to wrap the supporting cartilage block and was elevated above periosteum in rectangular shape, and then fixed with 4-0 vicryl sutures. Unlike in the study of Lim, however, we could elevate the flap with definitive anatomy and inevitable depression did not occur in our study.

Using only a skin flap among various surgical methods of auri- 
cle elevation after ear reconstruction in patients with congenital microtia, the degree of projection cannot be controlled and the three-dimensional structure cannot be maintained because the formation progresses after the elevation. On the other hand, if cartilage is used, the degree of projection can be controlled and the degree of contracture will not be that heavy. The elevated auricle tends to be become flat as time passes, we performed over-elevation by 5 degree than opposite auriculocephalic angle. But cartilage is vulnerable to infection, so aseptic surgical techniques and wound dressing after elevation must be provided.

Completing auricular reconstruction with two stage operation, it is important that the cartilage and soft tissue on the concha area would be removed to make enough deep concha area. If the concha was made deeply in the first stage operation, auricular elevation could make enough deep and good-looking concha. Of course, if the concha is not relatively deep after the second stage operation, deepening operation would be performed or canaloplasty could be performed according to patient's desire.

And we could decrease the secondary contracture of the skin graft by using FTSG instead of split-thickness skin graft. Although implanted cartilage may be absorbed some degree spontaneously but, the impact of the absorption could not be evaluated in this study. Complication rate was low and these complications were easily corrected in most of cases. We were able to reconstruct the competent ear, to maintain the three-dimensional shape and enough projection for a long time, and to give the patients satisfaction. Furthermore, our operation method was designed for the best time and economic efficiency with only twice of operations, and the results are mostly satisfying. Also, its gradual approach allows easy response to broad situations, which leads to less deformities and complications. It's a good strategy for gradual ear reconstruction.

\section{REFERENCES}

1. Beahm EK, Walton RL. Auricular reconstruction for microtia: part I. Anatomy, embryology, and clinical evaluation. Plast Reconstr Surg 2002;109:2473-82.

2. Lee TJ, Shin YJ, Lee YJ. Microtia correction using autogenous rib cartilage grafts- experience with 48 patients over a 7-year period. J Korean Soc Plast Reconstr Surg 1992;19:837-46.

3. Walton RL, Beahm EK. Auricular reconstruction for microtia: Part II. Surgical techniques. Plast Reconstr Surg 2002;110:234-49.

4. Kim JY, Cho BC, Lee SH. Two-stage ear reconstruction with canaloplasty in congenital microtia. J Korean Soc Plast Reconstr Surg 2006;33:53-60

5. Chun YH, Hyon WS, Ha BJ, Oh KS. Ear elevation using the superficial temporal fascia harvested through minimal incision. J Korean Soc Plast Reconstr Surg 2002;29:357-62.

6. Osorno G. A 20-year experience with the Brent technique of auricular reconstruction: pearls and pitfalls. Plast Reconstr Surg 2007;119:1447-63.

7. Nakai H. Reconstruction of microtia. Pursuing a natural appearance. Clin Plast Surg 1990;17:287-304.

8. Nagata S. Modification of the stages in total reconstruction of the auricle: Part IV. Ear elevation for the constructed auricle. Plast Reconstr Surg 1994;93:254-66.

9. Steffensen WH. A method of total ear reconstruction. Plast Reconstr Surg 1965;36:97-100.

10. Rueckert F, Brown FE, Tanzer RC. Overview of experience of Tanzer's group with microtia. Clin Plast Surg 1990;17:223-40.

11. Alexander G, Rajacic N, Ibrahim MK, Al-Zamil S. The combined posterior temporoparietal and galeal fascial flap: a new flap in the elevation of the constructed auricle (second stage of microtia correction). Br J Plast Surg 2002;55:582-4.

12. Brent B. Auricular repair with autogenous rib cartilage grafts: two decades of experience with 600 cases. Plast Reconstr Surg 1992;90:355-74.

13. Ou LF, Yan RS, Tang YW. Firm elevation of the auricle in reconstruction of microtia with a retroauricular fascial flap wrapping an autogenous cartilage wedge. Br J Plast Surg 2001;54:573-80.

14. Prantl L, Ashary I, Eisenmann-Klein M, Schwarze H. Modification of the second stage of auricular elevation in Nagata's technique for treating microtia. Scand J Plast Reconstr Surg Hand Surg 2007;41:109-13.

15. Yoshimura K, Nakatsuka T, Ichioka S, Kaji N, Harii K. One-stage reconstruction of an upper part defect of the auricle. Aesthetic Plast Surg 1998:22:352-5.

16. Lim SY, Mun GH, Hyon WS, Bang SI, Oh KS. The elevation of the constructed auricle with a temporoparietal fascial flap wrapping a resorbable plate. J Plast Reconstr Aesthet Surg 2006;59:505-9. 